\title{
Computational complexity and influence of numerical precision on the results of intercriteria analysis in the decision making process
}

\author{
Vassia Atanassova and Olympia Roeva \\ Department of Bioinformatics and Mathematical Modelling \\ Institute of Biophysics and Biomedical Engineering, Bulgarian Academy of Sciences \\ 105 Acad. Georgi Bonchev Str., Sofia 1113, Bulgaria \\ e-mails: vassia.atanassova@gmail . com, olympia@biomed.bas.bg
}

Received: 10 February 2018

Accepted: 7 March 2018

\begin{abstract}
The present step from the research on InterCriteria Analysis (ICrA) discusses the issues of the computational complexity of the algorithm developed, and the influence which the numerical precision has on the results of its work. These questions are important from both theoretical, and practical point of view, especially in the context of the application of the method in support of the decision making process.
\end{abstract}

Keywords: Intercriteria analysis, Computational complexity, Numerical precision, Decision making.

2010 Mathematical Subject Classification: 03E72, 03D15.

\section{Introduction}

The proposal of the intuitionistic fuzzy sets based method of InterCriteria Analysis (ICrA) by Atanassov, Mavrov and Atanassova, presented in front of the 12th International Workshop on Intuitionistic Fuzzy Sets and Generalized Nets '2013 in Warsaw, Poland, and published in [4], was followed by intensive developments in both theoretical and application aspect. Under this method, arrays of data obtained by the measurement of many objects against many criteria are processed to calculate pairwise dependencies between each pair of criteria in the form of intuitionistic fuzzy pairs of values in the $[0 ; 1]$-interval, thus rendering account of uncertainty. 
The original motivation behind the method was in handling cases of multicriteria decision making, where measuring according to some of the criteria is slower or more expensive, which results in delaying or raising the cost of the overall process of decision making. A particular problem from the field of the petrochemical industry required reasonable elimination of these criteria, in order to achieve economy and efficiency. Later, the ICrA produced good results in multiple other areas like economics, medicine, ecology, and in applications of certain bioinspired metaheuristics, optimization algorithms and neural networks. A complete list of publications with results on ICrA can be accessed from [13].

In the present step of the research, two important issues will be discussed: the computational complexity of the algorithm developed, and the influence which the numerical precision has on the results of its work.

\section{Computational complexity of the ICrA algorithm}

Here we will briefly remind the reader of the framework of the proposed method, following the notation from [3] and onward. The input of the method requires an index matrix (see [1]) $M$ of dimension $m \times n$, containing of $m$ rows $\left\{O_{1}, \ldots, O_{m}\right\}$ and $n$ columns $\left\{C_{1}, \ldots, C_{n}\right\}$, where for every $p, q(1 \leq p \leq m, 1 \leq q \leq n), O_{p}$ is an evaluated object, $C_{q}$ is an evaluation criterion, and $e_{O_{p}} C_{q}$ is the evaluation of the $p$-th object against the $q$-th criterion:

$M=$\begin{tabular}{c|ccccc} 
& $C_{1}$ & $\ldots$ & $C_{k}$ & $\ldots$ & $C_{n}$ \\
\hline$O_{1}$ & $e_{O_{1}, C_{1}}$ & $\ldots$ & $e_{O_{1}, C_{k}}$ & $\ldots$ & $e_{O_{1}, C_{n}}$ \\
$\vdots$ & $\vdots$ & $\ddots$ & $\vdots$ & $\ddots$ & $\vdots$ \\
$O_{i}$ & $e_{O_{i}, C_{1}}$ & $\ldots$ & $e_{O_{i}, C_{k}}$ & $\ldots$ & $e_{O_{i}, C_{n}}$ \\
$\vdots$ & $\vdots$ & $\ddots$ & $\vdots$ & $\ddots$ & $\vdots$ \\
$O_{m}$ & $e_{O_{m}, C_{1}}$ & $\ldots$ & $e_{O_{m}, C_{k}}$ & $\ldots$ & $e_{O_{m}, C_{n}}$
\end{tabular}

The demanded output is an index matrix of dimension $n \times n$, giving the calculated dependences between each pair of the input criteria. As we will see, these calculated dependences that populate the output matrix are in the form of intuitionistic fuzzy pairs (see [5]), although for practical reasons it is also appropriate to return two matrices, one containing the membership parts of the intuitionistic fuzzy pairs, and the other containing the non-membership parts.

The evaluations $e_{O_{p} C_{q}}(1 \leq p \leq m, 1 \leq q \leq n)$ were originally ${ }^{1}$ defined as real numbers, comparable according to relation $R$ in the form $R\left(e_{O_{i} C_{k}}, e_{O_{j} C_{k}}\right)$ for each $i, j, k$, with the result being "less than", "greater than", or "equal". For the needs of our method, such pairwise comparisons are performed for every pair of different criteria on all evaluated objects. For example, if the logic of our solved problem requires us to fix the relation $R$ to be "greater than", then $R$ has a dual relation $\bar{R}$, which is true in the cases when relation $R$ is false, i.e. when the result of the comparison is "less than".

During the described procedure of exhaustive (brute-force) pairwise comparisons, the ICrA algorithm maintains three numerical counters: one for the number of cases when the relation $R$

\footnotetext{
${ }^{1}$ Later, the method was upgraded to work with input data in the form of intuitionistic fuzzy data [9, 23] and normalized data [2], and it is expected to work well with linguistic variables with introduced ordering.
} 
holds, one for the number of cases when the dual relation $\bar{R}$ holds, and one for all the rest cases, i.e. when the relation $R\left(e_{O_{i}} C_{k}, e_{O_{j}} C_{k}\right)$ has resulted in "equal". These three counters are then given intuitionistic fuzzy interpretations of membership, non-membership and uncertainty, respectively, after norming in the [0,1]-interval, and thus the measures of dependence between the pairs of criteria are formed, with the three terms introduced, positive consonance, negative consonance and dissonance.

Therefore, we have $n(n-1) / 2$ pairs of non-identical criteria $\left(C_{k}, C_{l}\right)$ that must be compared, and for each pair we must obtain $m(m-1) / 2$ numerical comparisons of the objects' evaluations. Hence, the ICrA procedure we perform a total number of $m(m-1) n(n-1) / 4$ pairwise numerical comparisons, i.e. elementary operations. Therefore, the complexity of the ICrA algorithm will be $O\left(m^{2} n^{2}\right)$, which is polynomial in $m n$.

We will further note that two different software applications have been developed for the ICrA method, by Mavrov [14, 15] and by Ikonomov [12], both being freely available from [13].

\section{Influence of the numerical precision}

ICrA is a completely data driven approach, which means that the numerical precision of input and output data is of critical importance for the precision of the decisions made on this basis. So far, a lot of research has been done in one of the most important aspects of the method, namely, the way the thresholds of consonance and dissonance are determined: as this is what gives the measure of precision of the decision made using ICrA. Setting the thresholds can, obviously, be a completely expert's decision, but an algorithmic approach is considered to yield more precision, objectivity and sustainability. This is why a series of papers has explored this issue in a stepwise manner, based on both our deepening understanding of the ICrA method, and observations made during the application of the method to various real-life datasets, with a detailed overview provided in [10].

The precision of the input data, however, is of no less importance, although it has not been explored in details so far.

While it is a matter of through investigation with various datasets and over different problems, we will here present the findings from an application of ICrA over analysis of model parameters correlations of an E. coli fed-batch cultivation process, on the one hand, and between genetic algorithms autosomes (total computational time and decision accuracy) during model parameters identification procedures, on the other hand.

\subsection{Case study: parameter estimation of an $E$. coli fed-batch cultivation process using genetic algorithm}

\section{E. coli fed-batch cultivation process model}

The application of the general state space dynamical model to the E. coli fed-batch cultivation process leads to the following non-linear differential equation system [26]:

$$
\frac{d X}{d t}=\mu_{\max } \frac{S}{k_{S}+S} X-\frac{F}{V} X
$$




$$
\begin{gathered}
\frac{d S}{d t}=-\frac{1}{Y_{S / X}} \mu_{\max } \frac{S}{k_{S}+S} X+\frac{F}{V}\left(S_{i n}-S\right) \\
\frac{d V}{d t}=F
\end{gathered}
$$

where $X$ is the biomass concentration, [g/l]; $S$ is substrate concentration, [g/l]; $F$ is influent flow rate, $[1 / \mathrm{h}] ; V$ is bioreactor volume, $[1] ; S_{\text {in }}$ is influent glucose concentration, $[\mathrm{g} / \mathrm{l}] ; \mu_{\max }$ is maximum specific growth rate, $[1 / \mathrm{h}] ; Y_{S / X}$ is yield coefficient, $[\mathrm{g} / \mathrm{g}] ; k_{s}$ is saturation constants, $[\mathrm{g} / \mathrm{l}]$.

The model consists of a set of four differential Eqs. (1) - (3) thus represented: two dependent state variables $\mathbf{x}=[X S]$ and three unknown parameters $\mathbf{p}=\left[\begin{array}{lll}\mu_{\max } & k_{S} & Y_{S / X}\end{array}\right]$.

Parameter estimation problem of the presented non-linear dynamic system is stated as the minimization of the distance measure $J$ between the experimental and the model predicted values of the considered state variables:

$$
J=\sum_{i=1}^{n} \sum_{j=1}^{k}\left\{\left[\mathbf{y}_{\exp }(i)-\mathbf{y}_{\bmod }(i)\right]_{j}\right\}^{2} \rightarrow \min
$$

where $n$ is the length of the data vector for each state variable $k ; \mathbf{y}_{\exp }$ are known experimental data; $\mathbf{y}_{\text {mod }}$ are model predictions with a given set of the parameters $\mathbf{p}$.

The cultivation experiments are performed in the Institute of Technical Chemistry, University of Hannover, Germany during the collaboration work with the Institute of Biophysics and Biomedical Engineering, BAS, Bulgaria, granted by DFG. The cultivation conditions are presented in details in [17].

\section{Genetic algorithms}

Genetic algorithm (GA) is a model of machine learning which derives its behavior from a metaphor of the processes of evolution in nature [11]. Since their introduction and subsequent popularization, the GAs have been frequently used as an alternative optimization tool to the conventional methods and have been successfully applied to a variety of areas, and find increasing acceptance [16, 24, 26].

In Table 1, the GA parameters used in this work are presented. These settings are chosen on the basis of performed pre-test procedures.

\begin{tabular}{|l|r|}
\hline \multicolumn{1}{|c|}{ Genetic algorithm parameter } & \multicolumn{1}{c|}{ Value } \\
\hline generation gap & 0.97 \\
\hline crossover rate & 0.70 \\
\hline mutation rate & 0.05 \\
\hline precision of binary representation & 20 \\
\hline number of individuals & $5: 5: 200$ \\
\hline number of generations & 100 \\
\hline
\end{tabular}

Table 1. Genetic algorithm parameters 


\subsection{Intercriteria analysis}

\section{Input data}

40 different genetic algorithms with varying number of chromosomes in the populations, from 5 to 200, with a step of 5, have been run. Because of the stochastic characteristics of the applied algorithm, each GA has been run at least 30 times in order to carry out meaningful statistical analysis. The mean results of the model parameters estimates $\left(\mu_{\max }, k_{S}\right.$ and $\left.Y_{S / X}\right)$, total time for the solver to run $(T t)$ and objective function value $J$ (Eq. (4)) are considered as evaluation criteria as follows: $J$ value is $\mathrm{C} 1, T t-\mathrm{C} 2, \mu_{\max }-\mathrm{C} 3,, k_{S}-\mathrm{C} 4$, and $Y_{S / X}-\mathrm{C} 5$. Objects are the different GAs, i.e. different number of chromosomes in the population.

We will use these observation to investigate the influence of the numerical precision of the estimates on the ICrA results. We will compare the cases of two, three and four decimal digits of precision. The input dataset in its most complete form (four decimal digits of precision) is presented in Table 2 . The other two datasets are produced by means of rounding, respectively.

Table 2. Input data - estimates of the three model parameters and genetic algorithm total computational time and decision accuracy

\begin{tabular}{|r|c|c|c|c|c|}
\hline \multirow{2}{*}{$\begin{array}{c}\text { Object } \\
\text { ID }\end{array}$} & \multicolumn{5}{|c|}{ Criteria } \\
\cline { 2 - 6 } & $\mathbf{C 1}$ & $\mathbf{C 2}$ & $\mathbf{C 3}$ & $\mathbf{C 4}$ & $\mathbf{C 5}$ \\
\hline $\mathbf{5}$ & 5.3930 & 5.7460 & 0.5085 & 0.0157 & 2.0578 \\
\hline $\mathbf{1 0}$ & 5.6066 & 6.8204 & 0.5270 & 0.0208 & 2.0208 \\
\hline $\mathbf{1 5}$ & 5.3889 & 8.2192 & 0.5216 & 0.0190 & 2.0235 \\
\hline $\mathbf{2 0}$ & 4.5884 & 9.5207 & 0.4953 & 0.0136 & 2.0231 \\
\hline $\mathbf{2 5}$ & 4.6549 & 10.8925 & 0.4996 & 0.0143 & 2.0233 \\
\hline $\mathbf{3 0}$ & 4.6836 & 12.1764 & 0.5019 & 0.0148 & 2.0231 \\
\hline $\mathbf{3 5}$ & 4.6710 & 14.0349 & 0.5011 & 0.0146 & 2.0230 \\
\hline $\mathbf{4 0}$ & 4.5804 & 15.5881 & 0.4923 & 0.0128 & 2.0233 \\
\hline $\mathbf{4 5}$ & 4.5996 & 16.4529 & 0.4886 & 0.0121 & 2.0236 \\
\hline $\mathbf{5 0}$ & 4.5657 & 17.6858 & 0.4916 & 0.0128 & 2.0232 \\
\hline $\mathbf{5 5}$ & 4.5950 & 18.4164 & 0.4943 & 0.0133 & 2.0229 \\
\hline $\mathbf{6 0}$ & 4.5980 & 19.4315 & 0.4938 & 0.0132 & 2.0227 \\
\hline $\mathbf{6 5}$ & 4.6395 & 21.6774 & 0.4973 & 0.0139 & 2.0226 \\
\hline $\mathbf{7 0}$ & 4.6232 & 22.3123 & 0.4956 & 0.0134 & 2.0229 \\
\hline $\mathbf{7 5}$ & 4.5905 & 23.9254 & 0.4928 & 0.0130 & 2.0232 \\
\hline $\mathbf{8 0}$ & 4.5548 & 25.3658 & 0.4896 & 0.0123 & 2.0233 \\
\hline $\mathbf{8 5}$ & 4.5805 & 26.6039 & 0.4929 & 0.0130 & 2.0230 \\
\hline $\mathbf{9 0}$ & 4.5785 & 28.1259 & 0.4918 & 0.0128 & 2.0230 \\
\hline $\mathbf{9 5}$ & 4.5898 & 29.2268 & 0.4929 & 0.0129 & 2.0231 \\
\hline $\mathbf{1 0 0}$ & 4.5862 & 30.5544 & 0.4921 & 0.0128 & 2.0230 \\
\hline
\end{tabular}

\begin{tabular}{|c|c|c|c|c|c|}
\hline Object & \multicolumn{5}{|c|}{ Criteria } \\
\cline { 2 - 6 } ID & C1 & C2 & C3 & C4 & C5 \\
\hline 105 & 4.5781 & 32.2568 & 0.4898 & 0.0123 & 2.0232 \\
\hline 110 & 4.5522 & 34.1913 & 0.4871 & 0.0118 & 2.0228 \\
\hline 115 & 4.5651 & 34.7497 & 0.4873 & 0.0118 & 2.0233 \\
\hline 120 & 4.5492 & 36.1304 & 0.4864 & 0.0117 & 2.0229 \\
\hline 125 & 4.5445 & 37.5032 & 0.4887 & 0.0122 & 2.0227 \\
\hline 130 & 4.5498 & 38.8479 & 0.4900 & 0.0124 & 2.0228 \\
\hline 135 & 4.5687 & 40.5467 & 0.4913 & 0.0126 & 2.0227 \\
\hline 140 & 4.5543 & 41.5467 & 0.4894 & 0.0123 & 2.0230 \\
\hline 145 & 4.5368 & 43.4858 & 0.4849 & 0.0114 & 2.0231 \\
\hline 150 & 4.5558 & 44.8690 & 0.4890 & 0.0121 & 2.0225 \\
\hline 155 & 4.5295 & 45.7213 & 0.4878 & 0.0120 & 2.0221 \\
\hline 160 & 4.5643 & 47.7249 & 0.4914 & 0.0126 & 2.0226 \\
\hline 165 & 4.5472 & 48.6084 & 0.4871 & 0.0118 & 2.0226 \\
\hline 170 & 4.5226 & 50.5750 & 0.4881 & 0.0121 & 2.0226 \\
\hline 175 & 4.5388 & 51.8142 & 0.4883 & 0.0120 & 2.0228 \\
\hline 180 & 4.5391 & 53.6103 & 0.4873 & 0.0119 & 2.0229 \\
\hline 185 & 4.5481 & 54.3877 & 0.4896 & 0.0123 & 2.0226 \\
\hline 190 & 4.5365 & 55.8577 & 0.4866 & 0.0117 & 2.0229 \\
\hline 195 & 4.5438 & 57.8062 & 0.4856 & 0.0116 & 2.0227 \\
\hline $\mathbf{2 0 0}$ & 4.5419 & 58.9996 & 0.4888 & 0.0121 & 2.0226 \\
\hline
\end{tabular}

\section{Resultant ICrA matrices}

The results of the ICrA method applied on these input data are as given in Table 3 (for the input data with precision of four decimal digits, which is the maximally available), Table 4 (for the input data with precision of three decimal digits), Table 5 (for the input data with precision of two decimal digits), and Table 6 (for the input data with precision of one decimal digit). The computations in all the four cases were made with both developed software packages [12, 14], and yielded identical results per case. In each of the Tables 3-6, (a) stays for the membership parts of the intercriteria dependences, (b) stays for the table of the respective non-membership parts, and (c) goes for the calculated distances of the intercriteria points to the point $(1,0)$, i.e., the complete Truth, sorted in ascending way. Subsequently in Figure 1 (a)-(d), the results from 
the ICrA application on the four datasets are given graphically, with the intuitionistic fuzzy pairs plotted as points onto the intuitionistic fuzzy interpretational triangle $[6,7]$.

\begin{tabular}{|l|c|r|r|r|r|}
\hline $\boldsymbol{\mu}$ & \multicolumn{1}{c|}{$\boldsymbol{C}_{1}$} & \multicolumn{1}{c|}{$\mathbf{C}_{2}$} & $\mathbf{C}_{3}$ & $\mathbf{C}_{4}$ & $\mathbf{C}_{5}$ \\
\hline $\mathbf{C}_{1}$ & 1 & 0.141026 & 0.875641 & 0.853846 & 0.614103 \\
\hline $\mathbf{C}_{2}$ & 0.141026 & 1 & 0.187179 & 0.165385 & 0.253846 \\
\hline $\mathbf{C}_{3}$ & 0.875641 & 0.187179 & 1 & 0.961538 & 0.546154 \\
\hline $\mathbf{C}_{4}$ & 0.853846 & 0.165385 & 0.961538 & 1 & 0.541026 \\
\hline $\mathbf{C}_{5}$ & 0.614103 & 0.253846 & 0.546154 & 0.541026 & 1 \\
\hline
\end{tabular}

(a)

\begin{tabular}{|l|c|r|r|r|r|}
\hline $\mathbf{v}$ & $\mathbf{C}_{1}$ & \multicolumn{1}{c|}{$\mathbf{C}_{2}$} & $\mathbf{C}_{3}$ & $\mathbf{C}_{4}$ & $\mathbf{C}_{5}$ \\
\hline $\mathbf{C}_{1}$ & 0 & 0.858974 & 0.119231 & 0.114103 & 0.310256 \\
\hline $\mathbf{C}_{2}$ & 0.858974 & 0 & 0.807692 & 0.802564 & 0.670513 \\
\hline $\mathbf{C}_{3}$ & 0.119231 & 0.807692 & 0 & 0.006410 & 0.373077 \\
\hline $\mathbf{C}_{4}$ & 0.114103 & 0.802564 & 0.006410 & 0 & 0.358974 \\
\hline $\mathbf{C}_{5}$ & 0.310256 & 0.670513 & 0.373077 & 0.358974 & 0 \\
\hline
\end{tabular}

(b)

\begin{tabular}{|c|c|c|c|c|c|}
\hline$\mu$ & $C_{1}$ & $\mathrm{C}_{2}$ & $C_{3}$ & $\mathrm{C}_{4}$ & $C_{5}$ \\
\hline$C_{1}$ & 1 & 0.139744 & 0.848718 & 0.660256 & 0.180769 \\
\hline$C_{2}$ & 0.139744 & 1 & 0.158974 & 0.069231 & 0.066667 \\
\hline $\mathrm{C}_{3}$ & 0.848718 & 0.158974 & 1 & 0.742308 & 0.184615 \\
\hline $\mathrm{C}_{4}$ & 0.660256 & 0.069231 & 0.742308 & 1 & 0.380769 \\
\hline $\mathrm{C}_{5}$ & 0.180769 & 0.066667 & 0.184615 & 0.380769 & 1 \\
\hline
\end{tabular}

(a)

\begin{tabular}{|l|c|r|r|r|r|}
\hline $\mathbf{v}$ & $\mathbf{C}_{1}$ & \multicolumn{1}{c|}{$\mathbf{C}_{2}$} & \multicolumn{1}{c|}{$\mathbf{C}_{3}$} & $\mathbf{C}_{4}$ & $\mathbf{C}_{5}$ \\
\hline $\mathbf{C}_{1}$ & 0 & 0.857692 & 0.096154 & 0.030769 & 0.057692 \\
\hline $\mathbf{C}_{2}$ & 0.857692 & 0 & 0.788462 & 0.621795 & 0.169231 \\
\hline $\mathbf{C}_{3}$ & 0.096154 & 0.788462 & 0 & 0.001282 & 0.088462 \\
\hline $\mathbf{C}_{4}$ & 0.030769 & 0.621795 & 0.001282 & 0 & 0.074359 \\
\hline $\mathbf{C}_{5}$ & 0.057692 & 0.169231 & 0.088462 & 0.074359 & 0 \\
\hline
\end{tabular}

(b)

\begin{tabular}{|l|r|r|r|r|r|}
\hline $\boldsymbol{\mu}$ & \multicolumn{1}{c|}{$\mathbf{C}_{1}$} & $\mathbf{C}_{2}$ & $\mathbf{C}_{3}$ & $\mathbf{C}_{4}$ & $\mathbf{C}_{5}$ \\
\hline $\mathbf{C}_{1}$ & 1 & 0.141026 & 0.875641 & 0.853846 & 0.614103 \\
\hline $\mathbf{C}_{2}$ & 0.141026 & 1 & 0.187179 & 0.165385 & 0.253846 \\
\hline $\mathbf{C}_{3}$ & 0.875641 & 0.187179 & 1 & 0.961538 & 0.546154 \\
\hline $\mathbf{C}_{4}$ & 0.853846 & 0.165385 & 0.961538 & 1 & 0.541026 \\
\hline $\mathbf{C}_{5}$ & 0.614103 & 0.253846 & 0.546154 & 0.541026 & 1 \\
\hline
\end{tabular}

\begin{tabular}{|l|r|r|r|r|r|}
\hline \multicolumn{7}{|c|}{$(\mathrm{a})$} \\
\hline $\mathbf{v}$ & $\mathbf{C}_{1}$ & $\mathbf{C}_{2}$ & $\mathbf{C}_{3}$ & $\mathbf{C}_{4}$ & $\mathbf{C}_{5}$ \\
\hline $\mathbf{C}_{1}$ & 0 & 0.858974 & 0.119231 & 0.114103 & 0.310256 \\
\hline $\mathbf{C}_{2}$ & 0.858974 & 0 & 0.807692 & 0.802564 & 0.670513 \\
\hline $\mathbf{C}_{3}$ & 0.119231 & 0.807692 & 0 & 0.006410 & 0.373077 \\
\hline $\mathbf{C}_{4}$ & 0.114103 & 0.802564 & 0.006410 & 0 & 0.358974 \\
\hline $\mathbf{C}_{5}$ & 0.310256 & 0.670513 & 0.373077 & 0.358974 & 0 \\
\hline
\end{tabular}

(b)

\begin{tabular}{|l|l|c|c|r|}
\hline $\mathbf{C}_{\mathbf{i}}$ & $\mathbf{C}_{\mathbf{j}}$ & $\boldsymbol{\mu}$ & $\mathbf{v}$ & distance to $\mathbf{T}$ \\
\hline $\mathrm{C}_{3}$ & $\mathrm{C}_{4}$ & 0.961538 & 0.00641 & 0.038992 \\
\hline $\mathrm{C}_{1}$ & $\mathrm{C}_{3}$ & 0.875641 & 0.119231 & 0.172282 \\
\hline $\mathrm{C}_{1}$ & $\mathrm{C}_{4}$ & 0.853846 & 0.114103 & 0.185419 \\
\hline $\mathrm{C}_{1}$ & $\mathrm{C}_{5}$ & 0.614103 & 0.310256 & 0.495152 \\
\hline $\mathrm{C}_{4}$ & $\mathrm{C}_{5}$ & 0.541026 & 0.358974 & 0.582683 \\
\hline $\mathrm{C}_{3}$ & $\mathrm{C}_{5}$ & 0.546154 & 0.373077 & 0.587506 \\
\hline $\mathrm{C}_{2}$ & $\mathrm{C}_{5}$ & 0.253846 & 0.670513 & 1.003162 \\
\hline $\mathrm{C}_{2}$ & $\mathrm{C}_{3}$ & 0.187179 & 0.807692 & 1.145881 \\
\hline $\mathrm{C}_{2}$ & $\mathrm{C}_{4}$ & 0.165385 & 0.802564 & 1.157883 \\
\hline $\mathrm{C}_{1}$ & $\mathrm{C}_{2}$ & 0.141026 & 0.858974 & 1.214773 \\
\hline
\end{tabular}

(c)

Table 3. Results of application on ICrA on the dataset with precision of four decimal digits

\begin{tabular}{|c|c|c|c|r|}
\hline $\mathbf{C}_{\mathbf{i}}$ & $\mathbf{C}_{\mathbf{j}}$ & $\boldsymbol{\mu}$ & $\mathbf{v}$ & distance to $\mathbf{T}$ \\
\hline $\mathrm{C}_{1}$ & $\mathrm{C}_{3}$ & 0.848718 & 0.096154 & 0.179254 \\
\hline $\mathrm{C}_{3}$ & $\mathrm{C}_{4}$ & 0.742308 & 0.001282 & 0.257695 \\
\hline $\mathrm{C}_{1}$ & $\mathrm{C}_{4}$ & 0.660256 & 0.030769 & 0.341134 \\
\hline $\mathrm{C}_{4}$ & $\mathrm{C}_{5}$ & 0.380769 & 0.074359 & 0.623679 \\
\hline $\mathrm{C}_{3}$ & $\mathrm{C}_{5}$ & 0.184615 & 0.088462 & 0.820169 \\
\hline $\mathrm{C}_{1}$ & $\mathrm{C}_{5}$ & 0.180769 & 0.057692 & 0.82126 \\
\hline $\mathrm{C}_{2}$ & $\mathrm{C}_{5}$ & 0.066667 & 0.169231 & 0.948552 \\
\hline $\mathrm{C}_{2}$ & $\mathrm{C}_{4}$ & 0.069231 & 0.621795 & 1.119357 \\
\hline $\mathrm{C}_{2}$ & $\mathrm{C}_{3}$ & 0.158974 & 0.788462 & 1.152821 \\
\hline $\mathrm{C}_{1}$ & $\mathrm{C}_{2}$ & 0.139744 & 0.857692 & 1.214775 \\
\hline
\end{tabular}

(c)

Table 4. Results of application on ICrA on the dataset with precision of three decimal digits

\begin{tabular}{|l|l|l|l|r|}
\hline $\mathbf{C}_{\mathbf{i}}$ & $\mathbf{C}_{\mathbf{j}}$ & $\boldsymbol{\mu}$ & $\boldsymbol{v}$ & distance to $\mathbf{T}$ \\
\hline $\mathrm{C}_{4}$ & $\mathrm{C}_{5}$ & 0.902564 & 0.000000 & 0.097436 \\
\hline $\mathrm{C}_{3}$ & $\mathrm{C}_{4}$ & 0.719231 & 0.000000 & 0.280769 \\
\hline $\mathrm{C}_{3}$ & $\mathrm{C}_{5}$ & 0.624359 & 0.002564 & 0.375650 \\
\hline $\mathrm{C}_{1}$ & $\mathrm{C}_{3}$ & 0.470513 & 0.006410 & 0.529526 \\
\hline $\mathrm{C}_{1}$ & $\mathrm{C}_{4}$ & 0.221795 & 0.000000 & 0.778205 \\
\hline $\mathrm{C}_{1}$ & $\mathrm{C}_{5}$ & 0.125641 & 0.001282 & 0.874360 \\
\hline $\mathrm{C}_{2}$ & $\mathrm{C}_{5}$ & 0.000000 & 0.050000 & 1.001249 \\
\hline $\mathrm{C}_{2}$ & $\mathrm{C}_{4}$ & 0.000000 & 0.142308 & 1.010075 \\
\hline $\mathrm{C}_{2}$ & $\mathrm{C}_{3}$ & 0.029487 & 0.393590 & 1.047286 \\
\hline $\mathrm{C}_{1}$ & $\mathrm{C}_{2}$ & 0.111538 & 0.808974 & 1.201584 \\
\hline
\end{tabular}

(c)

Table 5. Results of application on ICrA on the dataset with precision of two decimal digit 


\begin{tabular}{|l|r|r|r|r|r|}
\hline$\mu$ & \multicolumn{1}{|c|}{$\boldsymbol{C}_{1}$} & \multicolumn{1}{c|}{$\boldsymbol{C}_{2}$} & \multicolumn{1}{c|}{$\boldsymbol{C}_{3}$} & \multicolumn{1}{c|}{$\boldsymbol{C}_{4}$} & \multicolumn{1}{c|}{$\boldsymbol{C}_{5}$} \\
\hline $\mathbf{C}_{1}$ & 1 & 0.024359 & 0.374359 & 0.374359 & 0.420513 \\
\hline $\mathbf{C}_{2}$ & 0.024359 & 1 & 0 & 0 & 0 \\
\hline $\mathbf{C}_{3}$ & 0.374359 & 0 & 1 & 1 & 0.95 \\
\hline $\mathbf{C}_{4}$ & 0.374359 & 0 & 1 & 1 & 0.95 \\
\hline $\mathbf{C}_{5}$ & 0.420513 & 0 & 0.95 & 0.95 & 1 \\
\hline
\end{tabular}

(a)

\begin{tabular}{|r|r|r|r|r|r|}
\hline $\mathbf{v}$ & \multicolumn{1}{|c|}{$\mathbf{C}_{1}$} & \multicolumn{1}{c|}{$\mathbf{C}_{2}$} & $\mathrm{C}_{3}$ & $\mathrm{C}_{4}$ & \multicolumn{1}{c|}{$\mathbf{C}_{5}$} \\
\hline $\mathbf{C}_{1}$ & 0 & 0.601282 & 0 & 0 & 0.001282 \\
\hline $\mathbf{C}_{2}$ & 0.601282 & 0 & 0 & 0 & 0.05 \\
\hline $\mathrm{C}_{3}$ & 0 & 0 & 0 & 0 & 0 \\
\hline $\mathbf{C}_{4}$ & 0 & 0 & 0 & 0 & 0 \\
\hline $\mathrm{C}_{5}$ & 0.001282 & 0.05 & 0 & 0 & 0 \\
\hline
\end{tabular}

(b)

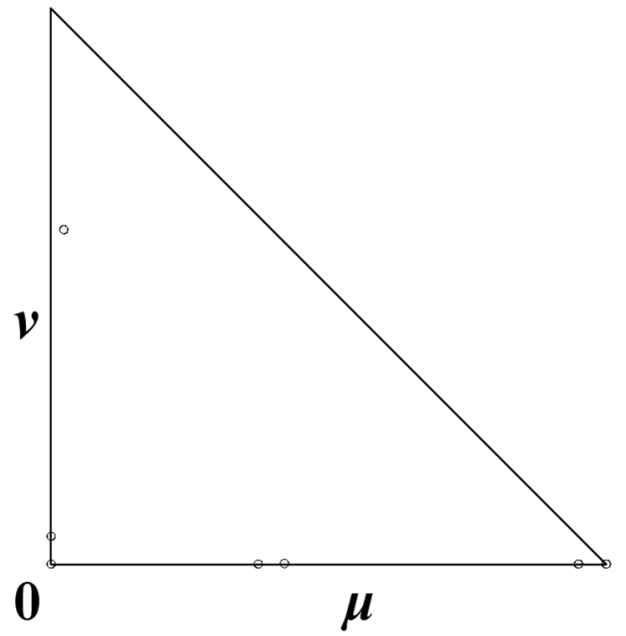

(a)

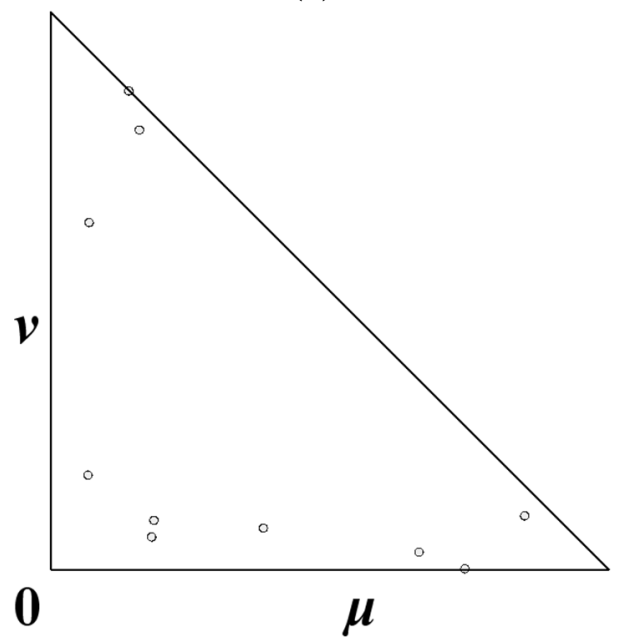

(c)

\begin{tabular}{|l|l|r|r|r|}
\hline $\mathbf{C}_{\mathbf{i}}$ & $\mathbf{C}_{\mathbf{j}}$ & \multicolumn{1}{|c|}{$\boldsymbol{\mu}$} & $\mathbf{v}$ & distance to $\mathbf{T}$ \\
\hline $\mathrm{C}_{3}$ & $\mathrm{C}_{4}$ & 1 & 0 & 0 \\
\hline $\mathrm{C}_{3}$ & $\mathrm{C}_{5}$ & 0.95 & 0 & 0.05 \\
\hline $\mathrm{C}_{4}$ & $\mathrm{C}_{5}$ & 0.95 & 0 & 0.05 \\
\hline $\mathrm{C}_{1}$ & $\mathrm{C}_{5}$ & 0.420513 & 0.001282 & 0.579489 \\
\hline $\mathrm{C}_{1}$ & $\mathrm{C}_{3}$ & 0.374359 & 0 & 0.625641 \\
\hline $\mathrm{C}_{1}$ & $\mathrm{C}_{4}$ & 0.374359 & 0 & 0.625641 \\
\hline $\mathrm{C}_{2}$ & $\mathrm{C}_{3}$ & 0 & 0 & 1 \\
\hline $\mathrm{C}_{2}$ & $\mathrm{C}_{4}$ & 0 & 0 & 1 \\
\hline $\mathrm{C}_{2}$ & $\mathrm{C}_{5}$ & 0 & 0.05 & 1.001249 \\
\hline $\mathrm{C}_{1}$ & $\mathrm{C}_{2}$ & 0.024359 & 0.601282 & 1.146043 \\
\hline
\end{tabular}

(c)

Table 6. Results of application on ICrA on the dataset with precision of one decimal digit

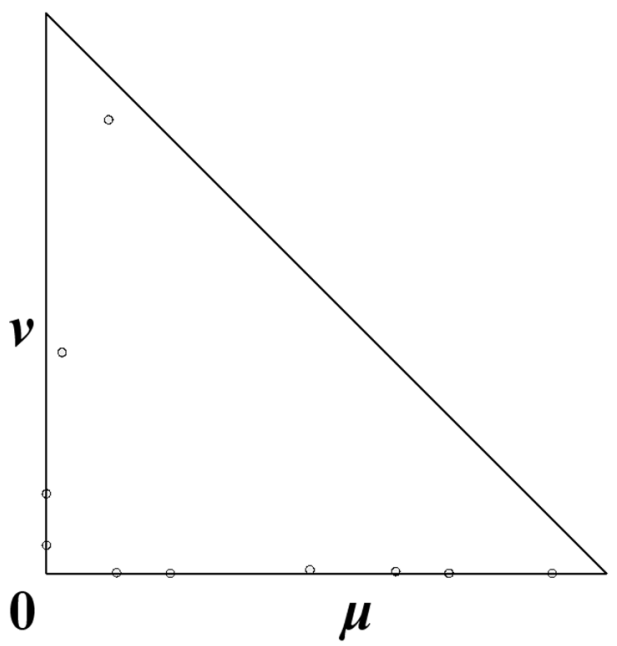

(b)

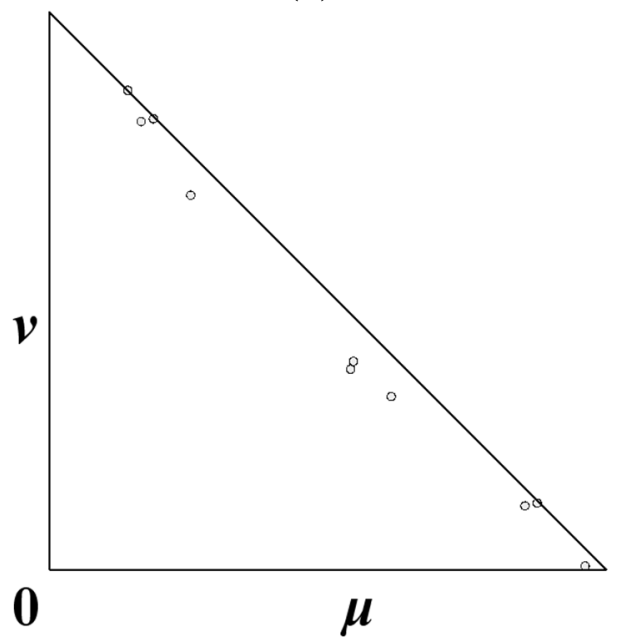

(d)

Figure 1. ICA results from Tables 3-6, plotted on the IFS triangle

\section{Discussion}

In the example discussed, we can see that some criteria pairs exhibit different behavior in considered cases (precision of four, three, two and one decimal digits). The obtained results are analyzed based on the scale proposed in [3], which defines the consonance and dissonance between the criteria pairs (see Table 7). 


\begin{tabular}{|c|l|}
\hline$\mu$ & \multicolumn{1}{|c|}{ Meaning } \\
\hline$[0.00-0.05]$ & strong negative consonance SNC \\
\hline$(0.05-0.15]$ & negative consonance NC \\
\hline$(0.15-0.25]$ & weak negative consonance WNC \\
\hline$(0.25-0.33]$ & weak dissonance WD \\
\hline$(0.33-0.43]$ & dissonance D \\
\hline$(0.43-0.57]$ & strong dissonance SD \\
\hline$(0.57-0.67]$ & dissonance D \\
\hline$(0.67-0.75]$ & weak dissonance WD \\
\hline$(0.75-0.85]$ & weak positive consonance WPC \\
\hline$(0.85-0.95]$ & positive consonance PC \\
\hline$(0.95-1.00]$ & strong positive consonance SPC \\
\hline
\end{tabular}

Table 7. Definition of consonance and dissonance between each pair of criteria

Our assumption is that the most reliable results are these obtained based on the estimations with precision of four decimal digits - in the case where we have the most information.

Based on the scale in Table 7, the results are summarized in Table 8 altogether with comparison of the previously published results about the considered here pair relations. It should be mentioned that the results in $[19,20,22]$ are obtained based on different optimization algorithms (altogether GA, ACO, FA, SA, ACO-GA, ACO-FA, and GA-ACO [20], only ACO [21] and only GA [22]), with different number of objects (7 [20], 11 [21] and 14 [22] objects). The considered criteria are the same for all investigations.

\begin{tabular}{|c|c|c|c|c|c|c|c|}
\hline \multirow{2}{*}{$\begin{array}{c}\text { Criteria } \\
\text { pair }\end{array}$} & \multicolumn{4}{|c|}{ Decimal digits } & \multicolumn{3}{|c|}{ Previous results } \\
\cline { 2 - 8 } & 4 & 3 & 2 & 1 & {$[20]$} & {$[21]$} & {$[22]$} \\
\hline C1-C2 & NC & NC & NC & SNC & D & SNC & NC \\
\hline C1-C3 & PC & WPC & SD & D & PC & WPC & WD \\
\hline C1-C4 & PC & D & WNC & D & WPC & D & D \\
\hline C1-C5 & D & WNC & NC & D & WD & D & D \\
\hline C2-C3 & WNC & WNC & SNC & SNC & D & SD & SD \\
\hline C2-C4 & WNC & NC & SNC & SNC & D & SD & D \\
\hline C2-C5 & WD & NC & SNC & SNC & WD & WD & WD \\
\hline C3-C4 & SPC & WD & WD & SPC & PC & PC & PC \\
\hline C3-C5 & SD & WNC & D & PC & D & WPC & WPC \\
\hline C4-C5 & SD & D & PC & PC & SD & WNC & WD \\
\hline
\end{tabular}

Table 8. Intercriteria analysis results

For the pair C1-C2 only in [20] a dissonance is identified. In all other cases the observed dependence is negative consonance or strong negative consonance. The negative consonance is a logical result - for a large number of algorithm iterations (i.e., greater computation time $T t$ ) it is more likely to find a more accurate solution, i.e., smaller value of $J$. The result in [20] could be explained with the fact that in this case seven different algorithms are considered (GA, ACO, FA, SA, ACO-GA, ACO-FA and GA-ACO). Due to stochastic nature of the algorithms it is possible to find a solution with high accuracy even for a small number of iterations (i.e., smaller $T t$ ), as an 
exception of the expectation that greater $T t$ lead to smaller $J$. Considering the results in the current research based on GA outcomes ( 40 objects) negative consonance is observed. The result is stable for different precision of decimal digits. The pair is in negative consonance independently from variation of the precision of decimal digits.

In case of precision of four decimal digits for the pairs $\mathrm{C} 1-\mathrm{C} 3, \mathrm{C} 1-\mathrm{C} 4$ and $\mathrm{C} 1-\mathrm{C} 5$ positive consonance, weak positive dissonance and weak dissonance are observed, respectively. According to [18] the parameters $\mu_{\max }$ and $k_{S}$ have higher sensitivity compared to parameter $Y_{S / X}$. Logically these parameters should be in consonance with the criterion value $J$. Moreover, taking into account the parameters sensitivity it is clear that the more sensitive parameter will be more linked to the value of $J$, and less sensitive parameter could show absence of correlation with $J$. The results in Table 7 show that for some cases even the most sensitive parameter may be in dissonance with $J$. So, the relations between the criteria pairs $\mathrm{C} 1-\mathrm{C} 3, \mathrm{C} 1-\mathrm{C} 4$ and $\mathrm{C} 1-\mathrm{C} 5$ are problem depended and for that reason they exhibit different behavior in different cases. This fact reflects on the results for different precision of decimal digits, but generally there are no contradictory results.

In case of precision of four decimal digits the pairs $\mathrm{C} 2-\mathrm{C} 3$ and $\mathrm{C} 2-\mathrm{C} 4$ are in weak negative dissonance and pair $\mathrm{C} 2-\mathrm{C} 5$ are in weak dissonance. For the other cases negative consonance (weak or strong) is observed for the pairs. Such results have not observed until now. It is difficult to define which results are more reliable. There is no enough data to decide if the smaller number of digits gives false results, or these criteria pairs could show very different behavior in different case problems. It is known that the total execution time is not dependent solely on one of the model parameters.

In case of precision of four and one decimal digits for the pair C3-C4 a SPC is observed. Considering the physical meaning of the considered model parameters [8] it is clear that there should be a strong correlation between these two criteria. The results presented in $[20,21,22]$ confirmed this expectation.

For the pair C3-C5 and C4-C5 a strong dissonance is observed. Considering the physical meaning of the model parameters [8] it is clear that there is a weak dependence between these two criteria (model parameters), as weak positive dissonance for the pair C3-C5 and weak negative dissonance for the pair C4-C5 [21]. Although it is possible in some particular cases to observe that there is no any relation between pairs $[20,22]$ and current research.

\section{Conclusion}

In this paper, two issues are discussed: the computational complexity of the InterCriteria Analysis algorithm and the influence which the numerical precision has on the results of its work. The complexity of the ICrA algorithm is $O\left(m^{2} n^{2}\right)$, which is polynomial in $m n$. Considering the influence of the numerical precision on the ICrA results, this investigation show that when the relation or dependence between two criteria is clearly expressed (in both directions - physically dependent or independent) this behavior is constantly expressed regardless of the precision of decimal digits. When the relation between two criteria is problem independent, i.e. specific conditions have the influence of the results. In this case, the criteria pairs behavior observed serve to analyze not only the relation between the criteria themselves, but mostly to analyze these specific conditions (in our case the behavior of metaheuristic algorithms). One more aspect is that the particular specifications of the ICrA approach also could manipulate the results according to 
precision of decimal digits. In case of greater precision of the input data, the chance for having the "equal" relation is smaller, which leads to less uncertainty in the results, hence more concreteness of the decision making process. On the other side, extremely high precision of the input may in certain cases also distort the results and prevent seeing "the big picture". So, adequate, comparable measures of precision are simultaneously needed at the input and at the output.

\section{Acknowledgements}

The present work is partially supported by the National Scientific Fund of Bulgaria under the Grant DN-I-02/10 and by the Program for Career Development of Young Scientists, Bulgarian Academy of Sciences, Grant DFNP-17-136/2017.

\section{References}

[1] Atanassov, K. (2014) Index Matrices. Springer, Cham.

[2] Atanassov, K., Atanassova, V., Chountas, P., Mitkova, M., Sotirova, E., Sotirov, S., \& Stratiev, D. (2016) Intercriteria analysis over normalized data. Proc. of 2016 IEEE 8th International Conference on Intelligent Systems, IEEE, 564-566.

[3] Atanassov, K., Atanassova, V., \& Gluhchev, G. (2015) InterCriteria analysis: ideas and problems. Notes on Intuitionistic Fuzzy Sets, 21(1), 81-88.

[4] Atanassov, K., Mavrov, D., \& Atanassova, V. (204) Intercriteria Decision Making: A New Approach for Multicriteria Decision Making, Based on Index Matrices and Intuitionistic Fuzzy Sets. Issues in Intuitionistic Fuzzy Sets and Generalized Nets, 11, 1-8.

[5] Atanassov, K., Szmidt, E., \& Kacprzyk, J. (2013) On intuitionistic fuzzy pairs. Notes on Intuitionistic Fuzzy Sets, 19(3), 1-13.

[6] Atanassova, V., Interpretation in the Intuitionistic Fuzzy Triangle of the Results, Obtained by the InterCriteria Analysis, 16th World Congress of the International Fuzzy Systems Association (IFSA), 9th Conference of the European Society for Fuzzy Logic and Technology (EUSFLAT), 30. 06-03. 07. 2015, Gijon, Spain, 2015, 1369-1374.

[7] Atanassova, V., Vardeva, I, Sotirova, E., \& Doukovska, L. (2016) Traversing and ranking of elements of an intuitionistic fuzzy set in the intuitionistic fuzzy interpretation triangle, Chapter, Novel Developments in Uncertainty Representation and Processing, Vol. 401, Advances in Intelligent Systems and Computing, 161-174.

[8] Bastin, G., \& Dochain, D. (1991) On-line Estimation and Adaptive Control of Bioreactors. Elsevier Science Publications.

[9] Bureva, V., Sotirova, E., Atanassova, V., Angelova, N., \& Atanassov, K. (2018) Intercriteria Analysis over Intuitionistic Fuzzy Data, Springer LNCS, Vol. 10655, 2018, 333-340.

[10] Doukovska L., Atanassova V., Sotirova E., Vardeva I., Radeva I. (2019) Defining Consonance Thresholds in InterCriteria Analysis: An Overview. In: Intuitionistic Fuzziness and Other Intelligent Theories and Their Applications. SCI, Vol 757. Springer, Cham. 
[11] Goldberg, D. E. (2006). Genetic Algorithms in Search, Optimization and Machine Learning. Addison Wesley Longman, London.

[12] Ikonomov, N., Vassilev, P., Roeva, O. (2018) ICrAData - Software for InterCriteria Analysis, Int J Bioautomation, 22(1), 1-10.

[13] Intercriteria.net. Available online: http://intercriteria.net/

[14] Mavrov, D. (2015) Software for InterCriteria Analysis: Implementation of the main algorithm, Notes on Intuitionistic Fuzzy Sets, 21(2), 77-86.

[15] Mavrov, D. (2015-2016) Software for intercriteria analysis:working with the results. Annual of "Informatics" Section, Union of Scientists in Bulgaria, 8, 37-44.

[16] Pencheva, T., Roeva, O., \& Angelova, M. (2018) Investigation of genetic algorithm performance based on different algorithms for intercriteria relations calculation. Lecture Notes in Computer Science, 10665, 390-398.

[17] Pencheva, T., Roeva, O., \& Hristozov, I. (2006) Functional State Approach to Fermentation, Processes Modelling, Prof. Marin Drinov Academic Publishing House, Sofia.

[18] Roeva, O. (2011) Sensitivity analysis of E. coli fed-batch cultivation local models. Mathematica Balkanica, New Series, 25(4), 395-411.

[19] Roeva, O., \& Fidanova, S. (2017) Intercriteria analysis of relations between model parameters estimates and ACO performance. Studies in Computational Intelligence, 681, $175-186$.

[20] Roeva, O., \& Fidanova, S. (2018) Comparison of different metaheuristic algorithms based on Intercriteria analysis. Journal of Computational and Applied Mathematics, 340, 615-628.

[21] Roeva O., S. Fidanova, M. Paprzycki, Comparison of Different ACO Start Strategies Based on InterCriteria Analysis, Recent Advances in Computational Optimization, Studies in Computational Intelligence, Vol. 717, 53-72.

[22] Roeva, O., Fidanova, S., Vassilev, P., \& Gepner, P. (2015) Intercriteria analysis of a model parameters identification using genetic algorithm. IEEE Proceedings of the 2015 Feder-ated Conference on Computer Science and Information Systems (FedCSIS), 5, 501-506

[23] Sekulić, M., Pejic, V., Brezocnik, M., Gostimirović, M., \& Hadžistević M. (2018) Prediction of surface roughness in the ball-end milling process using response surface methodology, genetic algorithms, and grey Wolf optimizer algorithm. Advances in Production Engineering \& Management, 13, 18-30.

[24] Traneva, V., Tranev, S., Szmidt, E. \& Atanassov, K. (2018) Three dimensional intercriteria analysis over intuitionistic fuzzy data. In: J. Kacprzyk et al. (eds.), Advances in Fuzzy Logic and Technology 2017, AISC, Vol. 641, Springer, 442-449.

[25] Zhang, Y., He, F., Lu, G., \& Xiong, H. (2018) An imporosity message scheduling based on modified genetic algorithm for time-triggered Ethernet. Science China, Information Sciences, 61(1), 019102.

[26] Zoteva, D., \& Roeva, O. (2018) InterCriteria analysis results based on different number of objects. Notes on Intuitionistic Fuzzy Sets, 24(1), 110-119. 